\title{
Efficacy of microneedling in treatment of acne scars
}

\author{
Essam A. Nada, Ramadan S. A Saleh and Mohammed A. Mostafa \\ Rasha I. Mohhmed \\ Department of Dermatology, Faculty of Medicine, Sohag University
}

\section{Abstract}

Introduction: Microneedling is a relatively new minimally invasive procedure involving superficial and controlled puncturing of the skin by rolling with miniature fine needles. Traditionally used as a collagen induction therapy for skin rejuvenation and facial scars specially acne scars.

Aim of the work: Evaluation of efficacy of microneedling in treatment of acne scars.

Patients and Methods: Ten patients with post acne atrophic facial scars attending the outpatient clinic of Dermatology in Sohag University Hospitals between June2014 to September 2017 were offered six sessions of microneedling at an interval of 1 month. They were evaluated monthly and one month after completion of sessions for both efficacy and safety of the procedure.

Results: The mean \pm SD of the patients' age in the study population were $26.900 \pm 5.952$ years old.eight females and two males with mean acne scars duration \pm SD $8.500 \pm 6.311$ years, $60 \%$ of patients had psychological distress from their acne scars. At the end of study duration Out of the ten patients 7(70\%) achieved one grade reduction in their Goodman and Baron qualitative grading score while 4 patients $(40 \%)$ achieved reduction in their quantitative ECCA " Echella d'e valuation Clinique des cicatrices d'acne" score with 8.55\% improvement in the ECCA score. The mean ECCA score value of V scar started to decrease earlier than U scar, but M scar was fixed up to end of sessions.

$50 \%$ of patients had good satisfaction, $30 \%$ of them had very good satisfaction and only $10 \%$ had excellent satisfaction. All treated patients, had adverse events of temporary erythema, edema, bleeding, or a serous ooze resolving with crusting or scabbing following each session of microneedling but in 1 patient (10\%) aggravation of acne occurred, bacterial infection not occurred in any of our patients.

Conclusion: Multiple minimally invasive sessions of skin microneedling is an effective treatment for post-acne atrophic scars with the advantage of being a relatively risk-free, inoffice procedure with minimal patient recovery time.

Key words: Acne, Scar, Microneedling.

\section{Introduction}

Acne vulgaris is a very common dermatological disease, it has a prevalence of over $90 \%$ among adolescents. Acne primarily affects areas of the skin with a relatively high number of sebaceous glands, including the face, upper part of the chest, and back. The resulting appearance can lead to anxiety, reduced self-esteem and, in extreme cases, depression or thoughts of suicide ${ }^{(1)}$. Inflammatory acne lesions can result in permanent scars, The prevalence and severity of acne scarring in the population has not been well studied, there was a study showed that nearly $1 \%$ of people had acne scars, although only 1 in 7 of these were considered to have "disfiguring scars". Acne scar estimated to affect $95 \%$ of people with acne vulgaris ${ }^{(1)}$

Inflammatory acne lesions can result in permanent scars ${ }^{(2)}$. The prevalence and severity of acne scarring in the population has not been well studied, although in the available literature is usually correlated to the severity of acne nearly $1 \%$ of people had acne scar (3)

There are two basic types of acne scar depending on whether there is a net loss or gain of collagen (atrophic and 
hypertrophic scars). Eighty to ninety percent of people with acne scars have scars associated with a loss of collagen (atrophic scars) compared to a minority who show hypertrophic scars and keloids ${ }^{(4)}$.

Severe scarring caused by acne is associated with substantial physical and psychological distress, particularly in adolescents ${ }^{(2)}$.

Despite high prevalence of acne scars, there is no single treatment modality that has been shown to be universally effective, posing a significant challenge for the treating physician. However, there are multiple methods for the treatment of acne scars available, each with both pros and cons (5)

Microneedling is a relatively new minimally invasive procedure involving superficial and controlled puncturing of the skin by rolling with miniature fine needles ${ }^{(6)}$. The needles also breakdown the old hardened scar strands and allow it to revascularize. Neovascularization and neocollagenesis is initiated by migration and proliferation of fibroblasts and laying down of intercellular matrix ${ }^{(7)}$. A fibronectin matrix forms after 5 days of injury that determines the deposition of collagen resulting in skin tightening persisting for 5-7 years in the form of collagen III. The depth of neocollagenesis has been found to be $5-600 \mu \mathrm{m}$ with a 1.5 $\mathrm{mm}$ length needle ${ }^{(8)}$. Histological examination of the skin treated with 4 microneedling sessions 1 month apart shows upto $400 \%$ increase in collagen and elastin deposition at 6 months postoperatively, with a thickened stratum spinosum and normal rete ridges at 1 year postoperatively. Collagen fibre bundles appear to have a normal lattice pattern rather than parallel bundles as in scar tissue ${ }^{(9)}$.
The numerous adverse effects and prolonged downtime associate energy based techniques make non energy based techniques like microneedling and topical agents are more suitable to wide range of acne scars sufferers ${ }^{(5)}$.

\section{Aim of the work:}

Evaluation of efficacy of microneedling in treatment of acne scars.

\section{Patients and Methods:}

Design: Cross sectional clinical study. Patients:

Ten patients with post acne atrophic facial scars attending the outpatient clinic of Dermatology in Sohag University Hospitals between $9^{\text {th }}$ June 2014 to $5^{\text {th }}$ September 2017 were offered six sessions of microneedling at an interval of 1 month.

The study was approved by the institute of research and ethical committees at Sohag faculty of medicine.

All the patients were informed verbally about the entire procedure, medication, possible complication and outcomes. An informed consent was signed from each participant. Patients were evaluated monthly and one month after completion of sessions for both efficacy and safety of the procedure.

\section{Methods:}

All patients were subjected to Medical history and Clinical examination including patients evaluation by Goodman and Baron grading system for qualitative description of post acne scar (10) and Echellad'e valuation Clinique des cicatrices d'acne (ECCA) scale for numerical grading of acne scars ${ }^{(11)}$. This evaluation is repeated at each session.

Goodman and Baron qualitative description of post acne scars grade acne scars to four grads macular, mild, moderate and sever. 
ECCA grading scales are based on semiquantitative weighted assessments of 6 types of acne scars, namely, Vshaped atrophic scars (icepick), Ushaped atrophic scars (boxcar), Mshaped atrophic scars (rolling), hypertrophic inflammatory scars, keloid scars, and superficial elastolysis.

\section{The procedure:}

The derma roller used is a plastic device with a $12-\mathrm{cm}$ handle that holds a drum-shaped cylinder at the end, similar to a small paint roller, $2 \mathrm{~cm}$ in diameter and $2 \mathrm{~cm}$ in width. The surface of the cylinder holds 192 titanium needles in eight rows. Each $0.25 \mathrm{~mm}$ in diameter and $1.5 \mathrm{~mm}$ length sterilized by UV/ Gamma rays. The area to be treated was anesthetized with topical anesthesia for 45 minutes to one hour. After preparation of the area with $1 \%$ Betadine solution. A sterile single user derma roller was used for the treatment. The instrument was rolled in horizontal, vertical, and oblique directions for 10-20 times on the treatment area. After treatments, the bleeding was controlled with light pressure with sterile gauze and application of ice packs was then done to the treatment area. The face was cleaned with sterile normal saline and application of fucidic acid ointment twice daily until the wounds were completely healed (between 5-7 days). There was mild to moderate facial erythema and or oedema for few days.

\section{Outcome assessments:}

At each session and one month after completion of sessions all patients are assessed clinically by Goodman and Baron qualitative grading system and ECCA score. Photographic documentation using identical camera setting, lighting and patient .Patient satisfaction: was evaluated at the end of study and was graded as one of four categories represents the \% of subjective improvement in the acne scars after treatment as compared with prior to treatment. [ Excellent $(100 \%$ $76 \%)$, Very Good $(51 \%-75 \%)$, good $(26 \%-50 \%)$ and poor $(0 \%-25 \%)]$.

Safety assessment for adverse effects and recovery times were recorded at each treatment visits and follow up visit (bleeding, erythema, oedema, scaling and crusting, dyschromia and aggravation of inflammatory acne) after each session was done.

\section{Inclusion criteria:}

Facial acne scars and age greater than 18 years.

\section{Exclusion criteria:}

Active inflammatory acne, active infection in the treatment area (e.g., herpes simplex and verrucae), melanoma or lesions suspected of malignancy, isotretinoin use in the past year, dermatoses (e.g., eczema and psoriasis), sunburn, anticoagulant therapy and systemic disease (diabetes, hypertention, collagen diseas or bleeding tendency).

\section{Results}

The mean \pm SD of the patients' age in the study population were $26.900 \pm 5.952$. Eight females and two males with mean acne scars duration \pm SD $8.500 \pm 6.311,60 \%$ of patients had psychological distress. Before starting sessions $30 \%, 40 \%$ and $30 \%$ of patients were grade 2, 3 and 4 Goodman and Baron grading system respectively and the mean \pm SD of their ECCA score was $76.00 \pm 42.45$.

One month after completion of sessions $70 \%$ of patients showed one grade reduction in their Goodma and Baron score.

The mean value of ECCA score started to decrease after $4^{\text {th }}$ session, 1 month after treatment there was significant difference in ECCA score compared to baseline ( $p$ value $=0.03$ ), with $8.55 \%$ of improvement in the ECCA score ( Table 1). 
SOHAG MEDICAL JOURNAL

Vol. 22 No.1 Jan 2018
Efficacy of microneedling in treatment of acne scars Rasha Ismail Mohamed.et al

Table 1: Changes in ECCA score in each group after each session

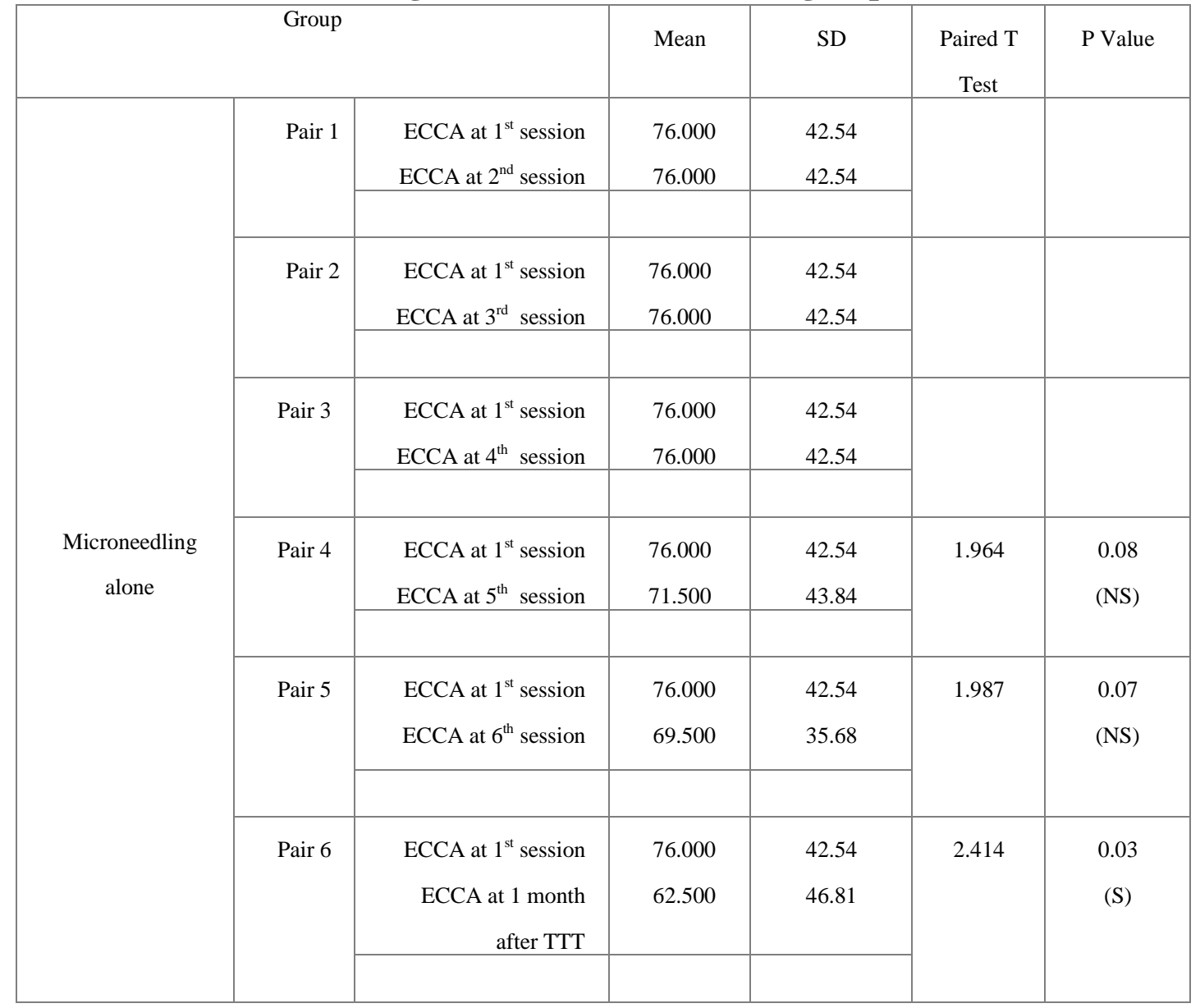

\section{$P$ value significant if $<0.05$}

The mean ECCA score value of $\mathrm{V}$ scar started to decrease after $3^{\text {rd }}$ session earlier than $\mathrm{U}$ scar that decreased after $4^{\text {th }}$ session, but $\mathrm{M}$ scar was fixed up to end of sessions. $50 \%$ of patients had good satisfaction, $30 \%$ of them had very good satisfaction and only $10 \%$ had excellent satisfaction.

All treated patients, had adverse events of temporary erythema, edema, bleeding, or a serous ooze resolving with crusting or scabbing following each session of microneedling but in 1 patient (10\%) aggravation of acne occurred, bacterial infection not occurred in any of our patients.

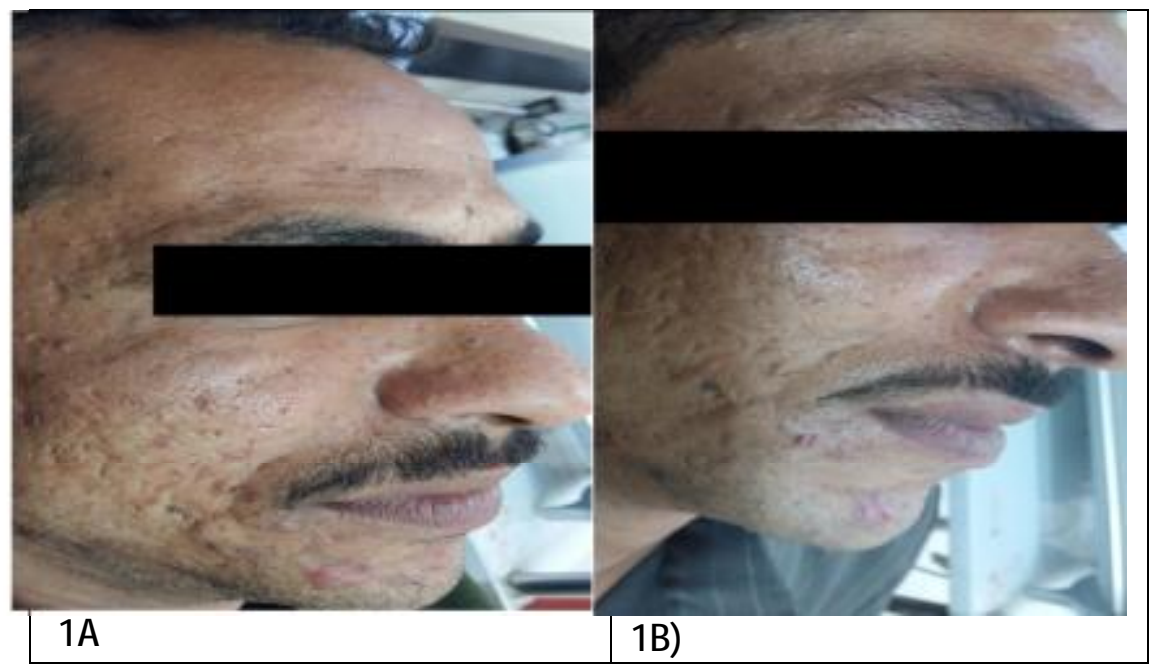




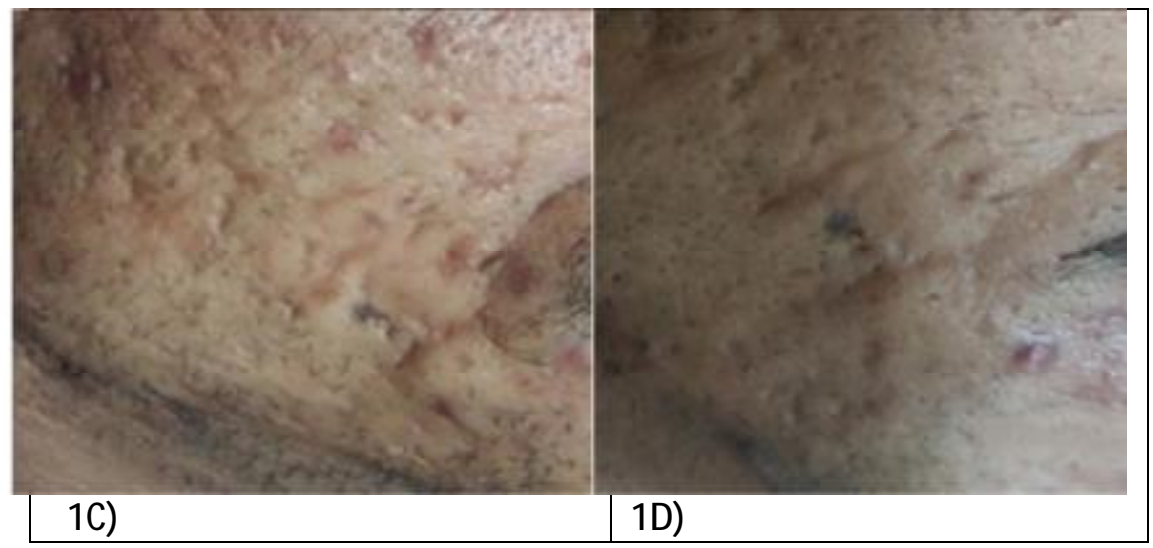

figure 1: received microneedling alone, right side of the face A) before treatment B) one month after six sessions $C$ ) close view before treatment D) close view after treatment.

\section{Discussion}

The challenge to compare our results to previous was due to heterogeneity in their sample size, study design and patient demographics. In addition, different scar grading scales have been used by different studies.

Our study population size was near to that conducted by Leheta et al. ${ }^{(12)}$, Sharad et al. (13), Gadkari and Nayak. (14), Leheta et al. ${ }^{(15)}$, El Domyati et al. ${ }^{(16)}$ to evaluate efficacy of microneedling either alone, compared to or in conjunction with other treatments of acne scars.

For microneedling we used the standard dermaroller device similar to that used in studies by Majid. (17), Chawla. ${ }^{(18)}$, Dogra et al. ${ }^{(19)}$, Leheta that used by Leheta et al. ${ }^{(15)}$, Nofal et al. ${ }^{(20)}$.

As regard to Goodman and Baron qualitative grading system we found; $70 \%$ of patients achieved one grade reduction in their grading system and $30 \%$ with no improvements. Our results were lower than that done by Asif et al. (21) that showed reduction by 2 grades in $10 \%$ of patients and by one grade in $84 \%$ of patients and also lower to study done by Majid. ${ }^{(17)}$ that showed reduction by two grades in $72.2 \%$ of patients and by one grade in $16,7 \%$ of patients. This can be et al. ${ }^{(15)}$, Nofal et al. ${ }^{(20)}$, El Domyati et al. ${ }^{(16)}$. Number of sessions was six sessions one every four weeks near to the average study durations and sessions intervals done by Majid. ${ }^{(17)}$, Dogra et al. ${ }^{(19)}$, Leheta et al. ${ }^{(15)}$, El Domyati et al. ${ }^{(16)}$ and more than that done by Chawla. ${ }^{(18)}$, Nofal et al. ${ }^{(20)}$.

For patients evaluation our study differ from all previous studies in combining subjective Goodman and Baron qualitative grading system as used by Majid. (17), Chawla. ${ }^{(18)}$, Nofal et al. (20) and objective numerical grading system Echelle d'Evaluation clinique des Cicatrices d'acne' classification (ECCA) scale that used only by Sharad. (13) and patient satisfaction

explained by the large number of patients involved in those two studies.

As regard to ECCA score, our study is the only one on microneedling used this quantitative score and our results showed that; the score improved in $40 \%$ of patients by the end of the study and the ECCA score began to decrease after the 4td session with a mean score \pm SD (71.5 \pm 43.84$)$ as compaired to the baseline $(76.00 \pm 42.54)$ and by the end of the study it was $(62.5 \pm 46.81) \quad$ with significant improvement by 13.5 points as compared to base line. The percent of 
improvement in the ECCA score in this group at the end of study was $8.55 \%$.

The mean value of $\mathrm{V}$ scar score started to decrease after 4th session, $U$ scar started to decrease after 5th session, but M scar was fixed up to end of sessions.

The improvement in types of scars in our study was in agree with the finding in a systematic review of treatments for acne scarring by Non-energy based techniques which done by Kravvas et al. (5) that noted that rolling scars have the highest rates of treatment failure

As regard patient satisfaction, there were only $10 \%$ of patients had excellent satisfaction, $30 \%$ had very good satisfaction and $50 \%$ of patients

\section{Conclusion:}

Multiple minimally invasive sessions of skin microneedling are an effective treatment for post-acne atrophic scars with the advantage of

\section{References:}

1. Admani S, Barrio VR. Evaluation and treatment of acne from infancy to preadolescence. Dermatologic therapy. 2013;26(6):462-6.

2. Fabbrocini G, Annunziata MC, D'Arco V, De Vita V, Lodi G, Mauriello MC, et al. Acne scars: pathogenesis, classification and treatment. Dermatology research and practice. 2010;2010:893080.

3. Cunliffe WJ, Gould DJ. Prevalence of facial acne vulgaris in late adolescence and in adults. British medical journal. 1979;1(6171):1109-10.

4. Midwood KS, Williams LV, Schwarzbauer JE. Tissue repair and the dynamics of the extracellular matrix. The international journal of biochemistry \& cell biology. 2004;36(6):1031-7. had good satisfaction. Patient satisfaction was not evaluated in studies on microneedling alone.

As regard adverse effects of the procedures:

Treated patients with skin needling alone, had adverse events of cause temporary erythema, edema, bleeding, or a serous ooze resolving with crusting or scabbing, the same result was reported by Fabbrocini et al. (9), Majid. (17), Fabbrocini et al. (22), Leheta et al. ${ }^{(12)}$, Sherad. ${ }^{(13)}$.

of acne is an uncommon adverse effect of microneedling as reported by Singh and Yadav. ${ }^{(23)}$, this side effect occurred in 1 patient (10\%). Similar to previous studies Harrris et al. (24), bacterial infection not occurred in any of our patients.

being a relatively risk-free, in-office procedure with minimal patient recovery time.

5. Kravvas G, Veitch D, Al-Niaimi F. The increasing relevance of biofilms in common dermatological conditions. The Journal of dermatological treatment. 2017:1-6.

6. Fernandes D. Minimally invasive percutaneous collagen induction. Oral and maxillofacial surgery clinics of North America. 2005; 17(1):51-63, vi.

7. Doddaballapur S. Microneedling with dermaroller. Journal of cutaneous and aesthetic surgery. 2009;2(2):110-1.

8. Bariya SH, Gohel MC, Mehta TA, Sharma OP. Microneedles: an emerging transdermal drug delivery system. The Journal of pharmacy and pharmacology. 2012;64(1):1129. 
9. Fabbrocini G, Fardella N, Monfrecola A, Proietti I, Innocenzi D. Acne scarring treatment using skin needling. Clinical and experimental dermatology. 2009;34(8):874-9.

10. Goodman GJ, Baron JA. Postacne scarring: a qualitative global scarring grading system. Dermatologic surgery : official publication for American Society for Dermatologic Surgery [et al]. 2006;32(12):1458-66.

11. Dreno B, Khammari A, Orain N, Noray C, Merial-Kieny C, Mery $\mathrm{S}$, et al. ECCA grading scale: an original validated acne scar grading scale for clinical practice in dermatology. Dermatology. 2007;214(1):46-51.

12. Leheta T, El Tawdy A, Abdel Hay R, Farid S. Percutaneous collagen induction versus fullconcentration trichloroacetic acid in the treatment of atrophic acne scars. Dermatologic surgery : official publication for American Society for Dermatologic Surgery [et al]. 2011;37(2):207-16.

13. Sharad J. Combination of microneedling and glycolic acid peels for the treatment of acne scars in dark skin. Journal of cosmetic dermatology. 2011;10(4):317-23.

14. Gadkari R, Nayak C. A splitface comparative study to evaluate efficacy of combined subcision and dermaroller against combined subcision and cryoroller in treatment of acne scars. Journal of cosmetic dermatology. 2014;13(1):38-43.

15. Leheta TM, Abdel Hay RM, El Garem YF. Deep peeling using phenol versus percutaneous collagen induction combined with trichloroacetic acid 20\% in atrophic post-acne scars; a randomized controlled trial. The Journal of dermatological treatment. 2014;25(2):130-6.

16. El-Domyati M, Barakat $\mathrm{M}$, Awad S, Medhat W, El-Fakahany H, Farag H. Microneedling Therapy for Atrophic Acne Scars: An Objective Evaluation. The Journal of clinical and aesthetic dermatology. 2015;8(7):36-42

17. Majid I. Microneedling therapy in atrophic facial scars: an objective assessment. Journal of cutaneous and aesthetic surgery. 2009;2(1):2630.

18. Chawla S. Split Face Comparative Study of Microneedling with PRP Versus Microneedling with Vitamin $\mathrm{C}$ in Treating Atrophic Post Acne Scars. Journal of cutaneous and aesthetic surgery. 2014;7(4):209-12.

19. Dogra S, Yadav S, Sarangal R. Microneedling for acne scars in Asian skin type: an effective low cost treatment modality. Journal of cosmetic dermatology. 2014;13(3):180-7.

20. Nofal E, Helmy A, Nofal A, Alakad R, Nasr M. Platelet-rich plasma versus CROSS technique with $100 \%$ trichloroacetic acid versus combined skin needling and platelet rich plasma in the treatment of atrophic acne scars: a comparative study. Dermatologic surgery : official publication for American Society for Dermatologic Surgery [et al]. 2014;40(8):864-73.

21. Asif M, Kanodia S, Singh K. Combined autologous platelet-rich plasma with microneedling verses microneedling with distilled water in the treatment of atrophic acne scars: a concurrent split-face study. 
Journal of cosmetic dermatology. 2016;15(4):434-43.

22. Fabbrocini G, De Vita V, Di Costanzo L, Pastore F, Mauriello MC, Ambra M, et al. Skin needling in the treatment of the aging neck. Skinmed. 2011;9(6):347-51.

23. Singh A, Yadav S.
Microneedling: Advances and widening horizons. Indian dermatology online journal. 2016;7(4):244-54.

24. Harris AG, Naidoo C, Murrell DF. Skin needling as a treatment for acne scarring: An up-to-date review of the literature. International journal of women's dermatology. 2015;1(2):77-81. 